\title{
Postradiation Osteosarcoma in an Older Prostate Cancer Survivor: Case Study and Literature Review with Emphasis on Geriatric Principles
}

\author{
Divya Gumber $^{a} \quad$ Miriam Rodin $^{a} \quad$ Tanya M. Wildes $^{b}$ \\ ${ }^{a}$ St. Louis University School of Medicine, and bashington University School of Medicine, \\ St. Louis, Mo., USA
}

\section{Key Words}

Osteosarcoma - Prostate cancer - Radiation therapy - Chemotherapy - Geriatrics · Elderly . Comorbidities $\cdot$ Comprehensive geriatric assessment

\begin{abstract}
The aging population and the increasing number of cancer survivors will likely be associated with more second primary malignancies due to prior cancer treatment. Since the incidence of most cancers increases with age, these treatment-associated second malignancies will likely disproportionately impact older adults. Here, we present the case of a 78-year-old man with a history of localized prostate cancer treated with external beam radiation therapy 11 years prior, who developed osteosarcoma of the ilium. Geriatric screening showed a fit older male with few comorbidities, functional independence and no other geriatric syndromes. Given the patient's preference for a limb-sparing operation, neoadjuvant chemotherapy was undertaken. With the paucity of clinical trial data on osteosarcoma in older adults, the patient was given a regimen of carboplatin (substituted for cisplatin), doxorubicin and methotrexate. Unfortunately, he developed methotrexate-induced acute kidney injury. Chemotherapy was discontinued, and he proceeded to hemipelvectomy. His postoperative course was marked by numerous complications, including delirium, depression and recurrent hospitalizations. He ultimately developed a local recurrence and elected for hospice care. This case highlights the challenges of managing older adults with treatment-associated malignancies. Clinicians face a lack of clinical trial data from which to extrapolate limitations of therapeutic options because of prior therapy and a limited ability to precisely predict which elders will experience adverse outcomes. Better approaches are needed to help older patients make decisions
\end{abstract}


which fulfill their goals of care and to improve the care of older adults with treatmentassociated malignancies.

\section{Introduction}

Cancer is a disease of aging, with more than $60 \%$ of new cancer cases and $70 \%$ of all cancer deaths occurring in patients older than 65 years of age [1]. Given the increasing life expectancy, a 67\% increase in cancer incidence in older patients is expected [2]. This will in turn result in an increasing number of older adult cancer survivors and an increase in longterm consequences of cancer therapy, including treatment-associated second malignancies.

Significant gaps in the evidence on treating older patients with cancer will bring challenges in clinical decision making. Elderly cancer patients are underrepresented in clinical trials, with only $25 \%$ of patients enrolled in clinical trials of chemotherapy over 65 years of age [3]. Underrepresentation of older adults in clinical trials may be due to explicit age restrictions or failure to meet eligibility criteria, but also relates to concerns that comorbidities may affect treatment response, concerns for difficulty adhering to study procedures, concerns about toxicity, or simply the attitude of physicians, patients and their families. Despite these perceptions, selected elderly patients may tolerate aggressive chemotherapy and derive as much benefit from them as their younger counterparts [4]. Our ability to reliably predict which elderly patients will do well and which will suffer severe toxicity is limited and is the subject of ongoing research.

The changing demographics, coupled with a limited evidence base on treating cancer in the elderly and the increasing risk of geriatric syndromes with advancing age, present challenges in treating this population. Here we present a case study of an older patient with a rare malignancy and highlight the challenges of treating cancer in older adults, spanning from initial treatment decision making to the end of life.

\section{Case Study}

The patient, a 78-year-old man with a history of a T1c, Gleason 8 adenocarcinoma of the prostate treated with external beam radiation to 7,000 Gy 11 years prior, presented to us with a slight increase in his prostate-specific antigen and pelvic pain. This prompted a bone scan, which demonstrated uptake in the left sacroiliac joint. A CT of the abdomen and pelvis demonstrated a mass in the lower pelvis extending from the prostate posteriorly and laterally through the obturator fossa into the obturator externus, but no distant metastases were found. Biopsy revealed osteosarcoma.

The patient's past medical history included deep venous thrombosis/pulmonary embolism incidentally diagnosed during staging evaluation, prostate cancer, hypertension, hyperlipidemia, asthma, and an episode of nephrotic syndrome 20 years prior. Geriatric screening at the time of his initial consultation with the medical oncologist revealed that he was independent in all of his activities of daily living (ADL) and instrumental activities of daily living (IADL). His ambulation was limited by pain in his pelvis. He had no symptoms of cognitive decline or depression. He reported a fall on icy pavement 5 months before presentation. He had no incontinence. His social support was extensive, including his wife, adult children, and grandchildren. Polypharmacy was noted, but he was on no medications considered inappropriate in the elderly by Beers criteria. 
His exam was only remarkable for bilateral lower extremity edema to the mid-shins. Laboratory values were notable for a creatinine level of $1.5 \mathrm{mg} / \mathrm{dl}$, with an estimated creatinine clearance of $39 \mathrm{ml} / \mathrm{min}$. His hemoglobin level was $10.8 \mathrm{~g} / \mathrm{dl}$. Creatinine clearance measured by a 24-hour urine collection was significantly higher than the calculated clearance $(79 \mathrm{ml} / \mathrm{min})$. Echocardiogram demonstrated diastolic dysfunction, but normal systolic function.

Multidisciplinary treatment planning was undertaken. The patient articulated that his primary goal of care would be to dance at his granddaughter's wedding in 3 months. As a first approach, surgical resection would require hemipelvectomy, a massive procedure which would have left the patient with only one leg and a large surgical pelvic defect. Neoadjuvant chemotherapy was proposed to downstage the tumor and potentially allow a limb-sparing resection. The patient tolerated his first cycle of chemotherapy with carboplatin and doxorubicin quite well, with improvement in his pelvic pain suggestive of response. He then proceeded with his first dose of methotrexate, which was empirically dose-reduced to $3.5 \mathrm{~g} / \mathrm{m}^{2}$ given concerns about his renal function. Unfortunately, despite the dose reduction, he developed acute renal failure and prolonged methotrexate clearance. He required hemodialysis for 3 weeks, ultimately recovering his renal function completely.

Restaging scans following the prolonged hospitalization demonstrated progression of the mass. The recommendation was to proceed with surgery. By this time, the family wedding was just a few weeks away, so surgery was delayed. The patient and his wife of over 50 years danced at the wedding.

The patient then underwent hemipelvectomy. Pathology demonstrated a large osteosarcoma with gross vascular invasion and residual tumor at the margins. His 2-week postoperative course was complicated by delirium. Subsequent to discharge, he was readmitted 1 week later with a hematoma. This was the first of 3 prolonged hospitalizations over the next 3 months with complications including gross hematuria, delirium, depression, pelvic abscess, urinary tract infection, atrial fibrillation, volume overload, and upper gastrointestinal bleeding.

Radiographic imaging 4 months after his surgery demonstrated a persistent large fluid collection within the pelvis. Biopsy demonstrated recurrent osteosarcoma. The patient and his family decided to focus on symptom management and enrolled in hospice. He died peacefully 2 weeks after his recurrence was diagnosed and 8 months after his initial diagnosis.

\section{Discussion}

This case of a radiation-associated osteosarcoma highlights challenges that will be faced with the increasing number of older adults with cancer, and of treating those malignancies in the face of little data while meeting the patient's goals of care.

\section{Prostate Cancer and Second Malignancies}

Prostate cancer is the most common cancer in men, with more than two thirds of the prostate cancer-related deaths occurring in men older than 75 years. Men treated with radiotherapy for prostate cancer are at increased risk for malignancies within the radiation field, including bladder, rectal and prostatic urethral cancer, with an absolute risk of $4.3 \%$ at 15 years of follow-up [5]. In the Surveillance, Epidemiology, and End Results Cancer Registry, patients with prostate cancer who underwent radiotherapy had a small but significantly increased risk of second malignancies, including cancers of the bladder and 
rectum as well as sarcomas within the radiation field. For patients who underwent radiation therapy, the relative risk of second malignancies was increased by $15 \%$ at 5 years and $34 \%$ at 10 years [6]. The median interval between radiation and development of a second malignancy was $10-15$ years [6].

\section{Osteosarcoma}

Osteosarcoma has a poorer prognosis in elderly patients as compared to younger patients; it is more likely to be diagnosed at a later stage, to be metastatic and to require limb amputation. The optimal management of osteosarcoma includes neoadjuvant chemotherapy followed by surgical resection with sufficiently wide surgical margins, and then additional adjuvant chemotherapy. With this approach, the 5-year disease-free survival rates have increased to more than $60 \%$, though these improvements have largely been limited to younger patients [7].

While radiation-associated sarcomas are long-term complications of various types of cancer, there are only rare reports after prostate cancer. However, with high-dose radiotherapy being widely utilized for the treatment of localized prostate adenocarcinoma, radiation-induced osteosarcoma should be considered for long-term survivors of prostate cancer who develop bone lesions within the prior radiation field [8].

\section{Treatment of Radiation-Associated Osteosarcoma in an Older Adult: Incorporation of \\ Geriatric Principles}

Deciding on the optimal treatment for this patient was challenging. Radiation therapy was not an option, given his previous radiation to the pelvis. Primary surgery was not initially acceptable to the patient, as it would have resulted in loss of mobility. Thus, neoadjuvant chemotherapy was felt to provide the best opportunity to downstage the tumor. However, to decide which chemotherapeutic regimen would provide optimal response while avoiding serious toxicity was a challenge. A systematic review and meta-analysis showed that a 3-drug regimen of adriamycin, cisplatin and methotrexate is associated with improved outcomes over 2-drug regimens [9]. Few patients in these studies were over the age of 40 years, let alone elderly. The decision was ultimately made to proceed with carboplatin, doxorubicin and high-dose methotrexate. Given concerns about toxicity with high-dose cisplatin in an older patient, a carboplatin-containing regimen was selected, based on a study in which the oldest patient was 24 years old [10].

Until recently, medical oncologists have had little data with which to counsel their older patients on their individual risk of toxicities of chemotherapy. Two recent studies have explored geriatric assessments as predictors of chemotherapy toxicity. In the Chemotherapy Risk Assessment Scale for High-Age Patients (CRASH) trial, adding subtle functional impairments identified with geriatric screening tools to the algorithm predicted toxicity better than standard oncology clinical and laboratory assessments alone. Specifically, dependence in IADL predicted hematologic toxicity, while Eastern Cooperative Oncology Group (ECOG) performance status, cognition and nutrition predicted nonhematologic toxicity [11]. In the Cancer and Aging Research Group (CARG) study, several domains of functional impairment identified with geriatric assessment screening tools were associated with toxicity of chemotherapy. These included hearing impairment, falls, difficulty taking medications (IADL), limitations in walking one block (IADL), and decreased social activity [12]. We note that although CARG and CRASH and other studies have also examined ADL screening tools, there appears to be a ceiling effect. That is, patients who are unable to perform their own self-care independently are less likely to present for treatment of advanced malignancy. Therefore, by evaluating IADL, the ability to live alone and maintain a 
household, we might detect more subtle impairments in patients who otherwise appear well. Our patient was at relatively lower risk based on these models. He tolerated the initial round of chemotherapy with carboplatin and doxorubicin quite well, but developed acute renal failure subsequent to methotrexate. While the multifactorial predictive models have certainly been an advance in the care of older adults, they are still imperfect prognostic tools. Our patient was functionally intact. Because of his known chronic kidney disease even with normal measured creatinine clearance, we reduced his methotrexate dose and chose a less toxic platinum agent. Nonetheless, severe renal toxicity due to chemotherapy started his decline.

The patient subsequently underwent hemipelvectomy, with a series of postoperative complications. There are several tools available to predict postoperative complications in older adults undergoing surgery. The Preoperative Assessment of Cancer in the Elderly (PACE) was created to evaluate the functional reserve of elderly cancer patients in need of surgery, and predict their postoperative morbidity and mortality [13]. PACE includes parameters of the Comprehensive Geriatric Assessment (CGA) such as functional status (IADL), depression and cognition, but also measures of fatigue, ECOG performance status, comorbidities and the degree of sickness prior to anesthesia and surgery (American Society of Anesthesiologists scale). Dependence in IADL, fatigue and abnormal performance status predicted postoperative complications and prolonged hospital stay. In another study, the geriatric syndrome of frailty was used. The phenotype of advanced age was defined as a score summarizing low grip strength (weakness), unintended weight loss, subjective exhaustion, low self-reported physical activity and slowed walking speed. Higher frailty scores predicted postoperative complications, length of stay and discharge to a nursing facility [14].

In the case presented here, the patient had been neither frail nor functionally impaired prior to chemotherapy, but by the time he presented for surgery, his strength, vitality and functional status had declined to the point that he was unable to perform his ADL independently. This decline was due both to his prolonged hospitalization for toxicity of chemotherapy and progression of the sarcoma.

\section{Conclusions}

In conclusion, with the aging of the population and increased incidence of cancer with age, clinicians will face an increasing number of older patients with primary and secondary malignancies. While there is a growing movement to increase our knowledge base on the optimal assessment and treatment of older patients with cancer, much remains to be learned. This case study encompasses many of the issues faced in caring for older adults, from second malignancies to treating a rare cancer in the absence of data, predicting toxicity or complications of treatment and meeting the patient's goals of care.

\section{Acknowledgements}

This publication was made possible by grant No. KM1CA156708 and 1K12CA167540 through the National Cancer Institute (NCI) at the National Institutes of Health (NIH) and grant No. UL1 TR000448 through the Clinical and Translational Science Award (CTSA) program of the National Center for Advancing Translational Sciences (NCATS) at the NIH. Its 
contents are solely the responsibility of the authors and do not necessarily represent the official view of NCI, NCATS or NIH.

\section{References}

-1 Yancik R, Ries LAG: Cancer in older persons: an international issue in an aging world. Sem Oncol 2004;31:128-136.

-2 Smith BD, Smith GL, Hurria A, Hortobagyi GN, Buchholz TA: Future of cancer incidence in the United States: burdens upon an aging, changing nation. J Clin Oncol 2009;27:2758-2765.

-3 Talarico L, Chen G, Pazdur R: Enrollment of elderly patients in clinical trials for cancer drug registration: a 7-year experience by the US Food and Drug Administration. J Clin Oncol 2004;22:4626-4631.

-4 Muss HB, Berry DA, Cirrincione CT, Theodoulou M, Mauer AM, Kornblith AB, et al: Adjuvant chemotherapy in older women with early-stage breast cancer. N Engl J Med 2009;360:2055-2065.

-5 Liauw SL, Sylvester JE, Morris CG, Blasko JC, Grimm PD: Second malignancies after prostate brachytherapy: incidence of bladder and colorectal cancers in patients with 15 years of potential follow-up. Int J Rad Oncol Bio Phys 2006;66:669-673.

6 Brenner DJ, Curtis RE, Hall EJ, Ron E: Second malignancies in prostate carcinoma patients after radiotherapy compared with surgery. Cancer 2000;88:398-406.

7 Bacci G, Picci P, Ferrari S, Ruggieri P, Casadei R, Tienghi A, et al: Primary chemotherapy and delayed surgery for nonmetastatic osteosarcoma of the extremities. Results in 164 patients preoperatively treated with high doses of methotrexate followed by cisplatin and doxorubicin. Cancer 1993;72:3227-3238.

8 Papalas JA, Wylie JD, Vollmer RT: Osteosarcoma after radiotherapy for prostate cancer. Ann Diag Path 2011;15:194-197.

-9 Anninga JK, Gelderblom H, Fiocco M, Kroep JR, Taminiau AHM, Hogendoorn PCW, et al: Chemotherapeutic adjuvant treatment for osteosarcoma: where do we stand? Eur J Canc 2011;47:2431-2445.

10 Daw NC, Neel MD, Rao BN, Billups CA, Wu J, Jenkins JJ, et al: Frontline treatment of localized osteosarcoma without methotrexate: results of the St. Jude Children's Research Hospital OS99 trial. Cancer 2011;117:2770-2778

11 Extermann M, Boler I, Reich RR, Lyman GH, Brown RH, DeFelice J, et al: Predicting the risk of chemotherapy toxicity in older patients: the Chemotherapy Risk Assessment Scale for High-Age Patients (CRASH) score. Cancer 2012;118:3377-3386.

12 Hurria A, Togawa K, Mohile SG, Owusu C, Klepin HD, Gross CP, et al: Predicting chemotherapy toxicity in older adults with cancer: a prospective multicenter study. J Clin Oncol 2011;29:3457-3465.

13 Pope D, Ramesh H, Gennari R, Corsini G, Maffezzini M, Hoekstra HJ, et al: Pre-operative assessment of cancer in the elderly (PACE): a comprehensive assessment of underlying characteristics of elderly cancer patients prior to elective surgery. Surg Oncol 2006;15:189-197.

14 Makary MA, Segev DL, Pronovost PJ, Syin D, Bandeen-Roche K, Patel P, et al: Frailty as a predictor of surgical outcomes in older patients. J Am Coll Surg 2010;210:901-908. 